\title{
Platelet Satellitism Assessment
}

National Cancer Institute

\section{Source}

National Cancer Institute. Platelet Satellitism Assessment. NCI Thesaurus. Code

C116209.

The determination of the presence of platelet satellitism in a sample. 